\title{
Time-Dependent Evolving Null Horizons of a Dynamical Spacetime
}

\author{
K. L. Duggal \\ Department of Mathematics and Statistics, University of Windsor, Windsor, ON, Canada N9B 3P4 \\ Correspondence should be addressed to K. L. Duggal; yq8@uwindsor.ca
}

Received 20 November 2013; Accepted 19 December 2013; Published 22 January 2014

Academic Editors: U. Kulshreshtha and W.-H. Steeb

Copyright (C) 2014 K. L. Duggal. This is an open access article distributed under the Creative Commons Attribution License, which permits unrestricted use, distribution, and reproduction in any medium, provided the original work is properly cited.

\begin{abstract}
Totally geodesic null hypersurfaces have been widely used in the study of isolated black holes. In this paper, we introduce a new quasilocal notion of a family of totally umbilical null hypersurfaces called evolving null horizons (ENH) of a dynamical spacetime, satisfied under an appropriate energy condition. We focus on a variety of examples of ENHs and in some cases establish their relation with event and isolated horizons. We also present two specific physical models of an ENH in a black hole spacetime. Beside the examples, for further study we propose two open problems on possible general existence of an ENH in a black hole spacetime and its canonical or unique existence. The results of this paper have ample scope of working on totally umbilical null hypersurfaces of Lorentzian and, in general, semiRiemannian manifolds.
\end{abstract}

\section{Introduction}

It is well-known that null hypersurfaces play an important roll in the study of black hole horizons. A black hole is a region of spacetime which contains a huge amount of mass compacted into an extremely small volume. Shortly after Einstein's first version of the theory of gravitation that was published in 1915, in 1916, Karl Schwarzschild computed the gravitational fields of stars using Einstein's field equations. He assumed that the star is spherical, gravitationally collapsed, and nonrotating. His solution is called a Schwarzschild solution which is an exact solution of static vacuum fields of the point-mass. Since then, considerable work has been done on black hole physics of asymptotically flat and timeindependent spacetimes. Such isolated black holes deal with the following concepts of event and isolated horizons.

1.1. Event Horizons. A boundary of a spacetime is called an event horizon, briefly denoted by EH, beyond which events cannot affect the observer. Note that an event horizon is intrinsically a global concept since its definition requires the knowledge of the whole spacetime to determine whether null geodesics can reach null infinity. EHs have played a key role and this includes Hawking's area increase theorem, black hole thermodynamics, black hole perturbation theory, and the topological censorship results. The most important family is the Kerr-Newman black holes. Moreover, an EH always exists in black hole asymptotically flat spacetime under a weak cosmic censorship condition. We refer Hawking's paper on "event horizon" [1], three papers of Hájićek's work [2-4] on "perfect horizons" (later called "nonexpanding horizons" by Ashtekar et al. [5]), and a paper by Galloway [6] in which he has shown that the null hypersurfaces which arise most naturally in spacetime geometry and general relativity, such as black hole event horizons, are in general $C^{0}$ but not $C^{1}$. Also Chruściel and his collaborators' papers include key use of EHs (see his review paper [7] on "Recent results in mathematical relativity" and several latest papers, in particular "No Hair" Theorems). However, an event horizon is too global to be useful in a number of physical situations ranging from quantum gravity to numerical relativity and to astrophysics. In particular, since it refers to infinity, it cannot be used in specially compact spacetime. Moreover, to actually locate a black hole one needs to know the full spacetime metric up to the infinite future. Even if one locates the event horizon, using it to calculate the physical parameters is extremely difficult. See Ashtekar-Krishnan [8] for more information on why the notion of an event horizon is inappropriate for a variety of physical situations. Therefore, attempts were made 
to find a quasilocal concept of a horizon which requires only minimum number of conditions to detect a black hole and study its properties. To achieve this objective, in a 1999 paper [5] Asktekar et al. introduced the following concept of isolated horizons.

1.2. Isolated Horizons. Before giving the definition of isolated horizons we recall some features of the intrinsic geometry of 3-dimensional null hypersurface, say $\mathscr{H}$, of a spacetime $(M, g)$, where the metric $g_{i j}$ has signature $(-,+,+,+)$. Denote by $q_{i j}=g_{i j}$ the intrinsic degenerate induced metric on $\mathscr{H}$ which is the pull back of $g_{i j}$, where an under arrow denotes the pullback to $\mathscr{H}$. Degenerate $q_{i j}$ has signature $(0,+,+)$ and does not have an inverse in the standard sense, but, in the weaker sense, it admits an inverse $q^{i j}$ if it satisfies $q_{i k} q_{j m} q^{k m}=$ $q_{i j}$. Using this, the expansion $\theta_{(\ell)}$ is defined by

$$
\theta_{(\ell)}=q^{i j} \nabla_{i} \ell_{j}
$$

where $\ell^{i}$ is a future-directed null normal to $\mathscr{H}$ and $\nabla$ is the Levi-Civita connection on $M$. The vorticity-free Raychaudhuri equation is given by

$$
\frac{d\left(\theta_{(\ell)}\right)}{d s}=-R_{i j} \ell^{i} \ell^{j}-\sigma_{i j} \sigma^{i j}-\frac{\theta^{2}}{2},
$$

where $\sigma_{i j}=\unrhd_{(i} \ell_{j)}-(1 / 2) \theta_{(\ell)} q_{i j}$ is the shear tensor, $s$ is a pseudoarc parameter such that $\ell$ is null geodesic, and $R_{i j}$ is the Ricci tensor of $M$. We say that two null normals $\ell^{i}$ and $\widetilde{\ell}^{i}$ belong to the same equivalence class $[\ell]$ if $\widetilde{\ell}^{i}=c \ell^{i}$ for some positive constant $c$. The following are three notions of isolated horizons, namely, nonexpanding, weakly, and stronger isolated horizons, respectively.

Definition 1. A null hypersurface $(\mathscr{H}, q)$ of a 4-dimensional spacetime $(M, g)$ is called a nonexpanding horizon $(\mathrm{NEH})$ if

(1) $\mathscr{H}$ has a topology $R \times S^{2}$;

(2) any null normal $\ell$ of $\mathscr{H}$ has vanishing expansion, $\theta_{(\ell)}=0$;

(3) all equations of motion hold at $\mathscr{H}$ and stress energy tensor $T_{i j}$ is such that $-T_{j}^{i} \ell^{j}$ is future-causal for any future-directed null normal $\ell^{i}$.

Condition (1) is a restriction on topology of $\mathscr{H}$ which guarantee that marginally trapped surfaces are related to a black hole spacetime. Condition (2) and the energy condition of (3) imply from the Raychaudhuri equation (2) that $\stackrel{T_{i j} e^{j}}{\longleftarrow}=0$ and $=\unrhd_{(i} \ell_{j)} \equiv \mathfrak{E}_{\ell} q_{i j}=0$ on $\mathscr{H}$, which further implies that the metric $q_{i j}$ is time-independent. Note that $\mathfrak{E}_{\ell} q_{i j}=0$ on $\mathscr{H}$ does not necessarily imply that $\ell$ is a Killing vector of the full metric $g_{i j}$. In general, there does not exist a unique induced connection on $\mathscr{H}$ due to degenerate $q_{i j}$. However, on an NEH, the property $\mathfrak{E}_{\ell} q_{i j}=0$ implies that the spacetime connection $\nabla$ induces a unique (torsion-free) connection, say $\mathscr{D}$, on $\mathscr{H}$ which is compatible with $q_{i j}$.
Definition 2. The pair $(\mathscr{H},[\ell])$ is called a weakly isolated horizon (WIH) if $\mathscr{H}$ is an NEH and each normal $\ell \in[\ell]$ satisfies

$$
\left(\mathfrak{£}_{\ell} \mathscr{D}_{i}-\mathscr{D}_{i} £_{\ell}\right) \ell^{i}=0 .
$$

Condition (3) implies that, in addition to the metric $q_{i j}$, the connection component $\mathscr{D}_{i} \ell^{j}$ is also time-independent for a WIH. Given a NEH, one can always have an equivalence class $[\ell]$ (which is not unique) of null normals such that $(\mathscr{H},[\ell])$ is a WIH. Ashtekar et al. [9] have discussed the issue of "Freedom of the choice of $\ell$ " in satisfying condition (3).

Definition 3. A WIH $(\mathscr{H},[\ell])$ is called an isolated horizon (IH) if the full connection $\mathscr{D}$ is time-independent, that is, if

$$
\left(\mathfrak{E}_{\ell} \mathscr{D}_{i}-\mathscr{D}_{i} \mathfrak{k}_{\ell}\right) V^{j}=0
$$

for arbitrary vector fields $V$ tangent to $\mathscr{H}$.

An $\mathrm{IH}$ is stronger notion of isolation as its condition (4) cannot always be satisfied by a choice of null normals. Isolated horizons are quasilocal and do not require the knowledge of the whole spacetime. The class of spacetimes having isolated horizons is quite big. Any Killing horizon which is topologically $R \times S^{2}$ is a trivial example of an isolated horizon. See Ashtekar and Krishnan [8], Ashtekar et al. [10], Lewandowski [11], and Gourgoulhon and Jaramillo [12] for examples and their physical use.

On the other hand, we know that the isolated horizons model specifically quasilocal equilibrium regimes of black hole spacetimes. However, in nature, black holes are rarely in equilibrium. This led to research on a quasilocal framework to describe the geometry of the surface of the dynamical black hole, not just at its equilibrium state. First attempt in this direction was made by Hayward [13], in 1994, using the framework of $(2+2)$-formalism, based on the notion of trapped surfaces. He proposed the following notion of future, outer, and trapping horizons (FOTH).

Definition 4. A future, outer, and trapped horizon (FOTH) is a three-manifold $\Sigma$, foliated by family of closed 2-surfaces such that (i) one of its future-directed null normal, say $\ell$, has zero expansion, $\theta_{(\ell)}=0$, (ii) the other null normal, $\mathbf{k}$, has negative expansion $\theta_{(\mathbf{k})}<0$, and (iii) the directional derivative of $\theta_{(\ell)}$ along $\mathbf{k}$ is negative; $\mathfrak{E}_{\mathbf{n}} \theta_{(\ell)}<0$.

$\Sigma$ is either spacelike or null for which $\theta_{(\mathbf{k})}=0$ and $£_{\mathbf{k}} \theta_{(\ell)}=$ 0 . Hayward [13] derived the following general laws of black hole dynamics.

(a) Zeroth Law. The total trapping gravity of a compact outer marginal surface has an upper bound, attained if and only if the trapping gravity is constant.

(b) First Law. The variation of the area form along an outer trapping horizon is determined by the trapping gravity and an energy flux.

After this, Ashktekar and Krishnan [14, 15] observed that in dynamical situations Hayward's condition (iii) is not 
required for most of the key physical results. For this reason, they introduced in [14] the following quasilocal concept of "dynamical horizons," denoted by DH, which model the evolving black holes and their asymptotic states are isolated horizons.

Definition 5. A smooth, 3-dimensional spacelike submanifold (possibly with boundary) $H$ of a spacetime is said to be a dynamical horizon (DH) if it can be foliated by a family of closed 2-manifolds such that

(1) on each leaf $S$ its future-directed null normal $\ell$ has zero expansion, $\theta_{(\ell)}=0$,

(2) and the other null normal, $\mathbf{k}$, has negative expansion $\theta_{(\mathbf{k})}<0$.

They first required that $H$ be spacelike everywhere and then studied the case in which portions of marginally trapped surfaces lie on a spacelike horizon and the remainder on a null horizon. In the null case, $H$ reaches equilibrium for which the shear and the matter flux vanish and this portion is represented by a weakly isolated horizon. The Vaidya metrics are explicit examples of dynamical horizons with their equilibrium states of the isolated horizons. The horizon geometry of DHs is time-dependent. Compared to Hayward's $(2+2)$ formalism, the $\mathrm{DH}$ framework is based on the standard $(1+$ 3)-formulism and has the advantage that it only refers to the intrinsic structure of $H$, without any conditions on the evolution of fields in transverse directions to $H$. In [15] Ashtekar and Krishnan have obtained the following main results.

(i) Expressions of fluxes of energy and angular momentum carried by gravitational waves across these horizons were obtained.

(ii) A detailed area balance law relating to the change in the area of $H$ to the flux of energy across it provided.

(iii) The cross sections $\mathcal{S}$ of $H$ have the topology $S^{2}$ if the cosmological constant (of Einstein's filed equations) is positive and of $S^{2}$ or a 2 -torus. The 2-torus case is degenerate as the matter and the gravitational energy flux vanish, the intrinsic metric on each $\mathcal{S}$ is flat, and the shear of the (expansion free) null normal vanishes.

(iv) A generalization of the first and the second laws of mechanics was obtained.

(v) A relation between DHs and $\mathrm{IH}$ was established.

$\mathrm{DH}$ has provided a new perspective covering all areas of black holes, that is, quantum gravity, mathematical physics, numerical relativity, and gravitational wave phenomenology, leading to the underlying unity of the subject.

As explained in [15], the definition of $\mathrm{DH}$ rules out the possibility of $H$ null, except when $H$ reaches equilibrium. Thus, we do not have any null horizon which is timedependent and quasilocal, and is always a null geodesic hypersurface. Such a null horizon is desired for information on the geometry and physics of the null surface of a dynamical spacetime, along with DHs which are models of evolving black holes. Moreover, there is a need to know the null version of the known results provided by FOTH and the DH frameworks. For these reasons, in this paper we study a new class of null horizons as explained below.

Observe that all types of null horizons (defined above) have a common condition that their future null normal has vanishing expansion. This means that their underlying null hypersurface is totally geodesic in the corresponding spacetime and these horizons are time-independent. In this paper, we use totally umbilical geometry to show the existence of a class of null hypersurfaces, called "Evolving Null Horizons (ENH)" (see Definition 8) (this notion is slightly general than recently introduced in [16] from where we take some material to present their improved version) which is time-dependent, quasilocal, and suitable for the null geometry of the surface of a dynamical spacetime. We focus on a variety of examples of ENHs, some of them having a totally geodesic null portion which represents an event or an isolated horizon at the equilibrium state of a black hole spacetime. This paper has been written with a twofold objective in mind firstly, initiate a new way of research on time-dependent null hypersurfaces of dynamical spacetimes by using the totally umbilical geometry. Secondly, the mathematical theory (using intrinsic geometry) on the foliations of null hypersurfaces presented in this paper (see also [16] for more general details) is expected to generate interest in further research on differential geometry of totally umbilical null hypersurfaces of Lorentzian and, in general, semiRiemannian manifolds.

\section{Totally Umbilical Null Hypersurfaces}

Recall that a hypersurface $(\mathscr{H}, h)$ of a 4 -dimensional spacetime $(M, g)$ is null if there exists a nonvanishing null vector field $\ell$ in $T(\mathscr{H})$ which is orthogonal (with respect to $h$ ) to all vector fields in $T(\mathscr{H})$; that is,

$$
h(\ell, X)=0, \quad \forall X \in T(\mathscr{H})
$$

where $h$ is the degenerate metric of $\mathscr{H}$. In this paper we assume that the null normal $\ell$ is future-directed and it is not entirely in $\mathscr{H}$ but is defined in some open subset of $M$ around $\mathscr{H}$. This will permit us to well define the spacetime covariant derivative $\nabla \ell$ and is suitable for the intrinsic geometry. For general extrinsic geometry of null hypersurfaces of semiRiemannian manifolds (where null normal is taken entirely in the hypersurface) we refer Duggal and Bejancu [17, Chapter 4] and Duggal and Jin [18, Chapter 7]. A simple way to take this extended $\ell$ is to consider a foliation of $M$ (in the vicinity of $\mathscr{H}$ ) by a family $\left(\mathscr{H}_{u}\right)$ so that $\ell$ is in the part of $M$ foliated by this family such that, at each point in this region, $\ell$ is a null normal to $\mathscr{H}_{u}$ for some value of $u$. Denote by $\left(h_{u}\right)$ the respective family of degenerate metrics. Although the family $\left(\mathscr{H}_{u}\right)$ is not unique, for our purpose we can manage (with some reasonable condition(s)) to involve only those quantities which are independent of the choice of the foliation $\left(\mathscr{H}_{u}\right)$ once evaluated at $\mathscr{H}_{u=\text { const. }}$. For simplicity, in this paper we consider $(\mathscr{H}, h)$ a member of the family $\left(\mathscr{H}_{u}\right)$ and its respective metric $h$ for some value of $u$, with the understanding that the results are the same for any other member. 
The "bending" of $\mathscr{H}$ in $M$ is described by the Weingarten map:

$$
\begin{aligned}
& \mathscr{W}_{\ell}: T_{p} \mathscr{H} \Longrightarrow T_{p} \mathscr{H}, \\
& X \Longrightarrow \nabla_{X} \ell .
\end{aligned}
$$

$\mathscr{W}_{\ell}$ associates each $X$ of $\mathscr{H}$ the variation of $\ell$ along $X$, with respect to the spacetime connection $\nabla$. The second fundamental form, say $B$, of $\mathscr{H}$ is the symmetric bilinear form and is related to the Weingarten map by

$$
B(X, Y)=h\left(\mathscr{W}_{\ell} X, Y\right)=h\left(\nabla_{X} \ell, Y\right) .
$$

$B(X, \ell)=0$ for any null normal $\ell$ and for any $X \in$ $T(\mathscr{H})$ imply that $B$ has the same $\ell$ degeneracy as that of the induced metric $h$. Hence, it is natural to study a class of null hypersurfaces such that $B$ is conformally equivalent to the metric $h$. Geometrically, this means that $(\mathscr{H}, h)$ is totally umbilical in $M$ if and only if there is a smooth function $f$ on $\mathscr{H}$ such that

$$
B(X, Y)=f h(X, Y), \quad \forall X, Y \in T(\mathscr{H}) .
$$

The above definition does not depend on particular choice of $\ell . \mathscr{H}$ is called proper totally umbilical if and only if $f$ is nonzero on entire $\mathscr{H}$. In particular, a portion of $\mathscr{H}$ is called totally geodesic if and only if $B$ vanishes, that is, if and only if $f$ vanishes on that portion of $\mathscr{H}$. From $(7), B(X, \ell)=0$ for any null normal $\ell$, and (8), we conclude that $\mathscr{H}$ is totally umbilical in $M$ if and only if, on each neighborhood $\mathcal{U}$ the conformal function $f$ satisfies

$$
\mathscr{W}_{\ell} X=f X, \quad \forall \text { non-null } X \in \Gamma(T \mathscr{H}) .
$$

The following result is important in the study of null hypersurfaces.

Proposition 6. Let $\left(\mathscr{H}_{u}\right)$ be a family of null hypersurfaces of a Lorentzian manifold. Then each member $(\mathscr{H}, h)$ of $\left(\mathscr{H}_{u}\right)$ is totally umbilical if and only if its null normal $\ell$ is a conformal Killing vector of the degenerate metric $h$.

Proof. Consider a member $(\mathscr{H}, h)$ of $\left(\mathscr{H}_{u}\right)$. Using the expression $\mathfrak{E}_{\ell} h(X, Y)=h\left(\nabla_{X} \ell, Y\right)+h\left(\nabla_{Y} \ell, X\right)$ and $B(X, Y)$ symmetric in the above equation we obtain

$$
B(X, Y)=\frac{1}{2} \mathfrak{E}_{\ell} h(X, Y), \quad \forall X, Y \in T(\mathscr{H}),
$$

which is well defined up to conformal rescaling (related to the choice of $\ell$ ). Suppose $\mathscr{H}$ is totally umbilical; that is, (8) holds. Using this in (10) we have $\mathfrak{E}_{\ell} h=2 f h$ on $\mathscr{H}$. Therefore, $\ell$ is conformal Killing vector of the metric $h$. Conversely, assume $\mathfrak{E}_{\ell} h=2 f h$ on $\mathscr{H}$. Then,

$$
\begin{aligned}
\mathfrak{E}_{\ell} h(X, Y) & =h\left(\nabla_{X} \ell, Y\right)+h\left(\nabla_{Y} \ell, X\right)=2 h\left(\nabla_{X} \ell, Y\right) \\
& =2 h\left(\mathscr{W}_{\ell} X, Y\right)=2 f h(X, Y),
\end{aligned}
$$

which implies that (9) holds so $\mathscr{H}$ is totally umbilical.
2.1. Normalization of $\ell$ and Projector onto $(\mathscr{H}, h)$. Due to degenerate metric $h$, there is no canonical way from the null structure alone of $\mathscr{H}$, to define a projector mapping II : $T_{p} M \rightarrow T_{p}(\mathscr{H})$. Thus to obtain normalized expressions for $\ell$ there is a need for some extrastructure on $M$. For this purpose, we consider a $(1+3)$-spacetime $(M, g)$. This assumes a thin sandwich of $M$ evolved from a spacelike hypersurface $\Sigma_{t}$ at a coordinate time $t$ to another spacelike hypersurface $\Sigma_{t+d t}$ at coordinate time $t+d t$ whose metric $g$ is given by

$$
g_{i j} d x^{i} d x^{j}=\left(-\lambda^{2}+|U|^{2}\right) d t^{2}+2 g_{a b} U^{a} d x^{b} d t+g_{a b} d x^{a} d x^{b},
$$

where $x^{0}=t$ and $x^{a}(a=1,2,3)$ are spatial coordinates of $\Sigma_{t}$ with $g_{a b}$ its 3-metric induced from $g, \lambda=\lambda\left(t, x^{1}, x^{2}, x^{3}\right)$ is the lapse function, and $U$ is a spacelike shift vector. The choice of $(1+3)$-spacetime comes from the works of Ashtekar and Krishnan [14, 15] and Gourgoulhon and Jaramillo [12] on dynamical and isolated horizons, respectively. The coordinate time vector $\mathbf{t}=\partial / \partial t$ is such that $g(d t, \mathbf{t})=1$. For the $(1+3)$ spacetime $M$, one can write

$$
\mathbf{t}=\lambda \mathbf{n}+U, \quad \text { with } \mathbf{n} \cdot U=0,
$$

where $\mathbf{n}$ is the future timelike unit vector field. The question is how to normalize $\ell$. In general, each spacelike hypersurface $\Sigma_{t}$ intersects the null hypersurface $\mathscr{H}$ on some 2-dimensional submanifold $\mathbf{S}_{t}$; that is, $\mathbf{S}_{t}=\mathscr{H} \cap \Sigma_{t}$. Consider a family $\mathscr{F}=$ $\left(\mathscr{H}_{u}\right)$ in the vicinity of $\mathscr{H}$ defined by

$$
\mathbf{S}_{t, u}=\mathscr{H}_{u} \cap \Sigma_{t},
$$

where $\mathbf{S}_{t, u=1}$ is an element of this family $\left(\mathbf{S}_{t, u}\right)$. Let $\mathbf{s} \in \Sigma_{t}$ be a unit vector normal to $S_{t}$ defined in some open neighborhood of $\mathscr{H}$. Taking $\left(\mathbf{S}_{t}\right)$ a foliation of $\mathscr{H}$, the coordinate $t$ can be used as a nonaffine parameter along each null geodesic generating $\mathscr{H}$. We normalize $\ell$ of $\mathscr{H}$ such that it is tangent vector associated with this parameterization of the null generators; that is,

$$
\ell^{i}=\frac{d x^{i}}{d t}
$$

This means that $\ell$ is a vector field "dual" to the 1 -form $d t$. Equivalently, the function $t$ can be regarded as a coordinate compatible with $\ell$; that is,

$$
g(d t, \ell)=\nabla_{\ell} t=1
$$

Based on this, it is easy to see that $\ell$ has the following normalization:

$\ell=\lambda(\mathbf{n}+\mathbf{s}), \quad$ where $\mathbf{s} \cdot s=1, \quad v \in T_{p}\left(\mathbf{S}_{t}\right) \Longleftrightarrow \mathbf{s} \cdot v=0$,

which implies that $\ell$ is tangent to $\mathscr{H}$ with the property of Lie dragging the family of surfaces $\left(\mathbf{S}_{t, u}\right)$.

As any $\mathscr{H}$ is defined by $u=$ a constant, the gradient $d u$ is its normal; that is,

$$
h(d u, X)=0 \Longleftrightarrow \forall X \in T(\mathscr{H}) .
$$


Thus, the 1 -form $\underline{\ell}$ associated with the null normal $\ell$ is collinear to $d u$, that is,

$$
\underline{\ell}=e^{\rho} d u
$$

where $\rho \in \mathscr{H}$ is a scalar field. Now the question is how to find some direction transverse to $\mathscr{H}$. We see from the normalized equation (17) that there are timelike and spacelike transverse directions $\mathbf{n}$ and $\mathbf{s}$ (as they both do not belong to $\mathscr{H}$ ), respectively, which are normal to the 2-dimensional spacelike submanifold $S_{t}$. Since we already have the outgoing null normal $\ell$ tangent to $\mathscr{H}$, we define a transversal vector field $\mathbf{k}$ of $T_{p} M$ not belonging to $\mathscr{H}$ expressed as another suitable linear combination of $\mathbf{n}$ and $\mathbf{s}$ such that it represents the light rays emitted in the opposite direction, called the ingoing direction, satisfying

$$
g(\ell, \mathbf{k})=-1, \quad g(\mathbf{k}, \mathbf{k})=0 .
$$

Using the normalization (17) of $\ell$ and (20) we get the following normalized expression of the null transversal vector $\mathbf{k}$ :

$$
\mathbf{k}=\frac{1}{2 \lambda}(\mathbf{n}-\mathbf{s}) \text {. }
$$

Since two null normals $\ell$ and $\tilde{\ell}$ of $\mathscr{H}$ belong to the same equivalence class $[\ell]$ if $\tilde{\ell}=c \ell$ for some positive constant $c$, it follows from (20) that with respect to change of $\ell$ to $\tilde{\ell}$ there is another $\tilde{\mathbf{k}}=(1 / c) \mathbf{k}$ satisfying (20). Now we define the projector onto $\mathscr{H}$ along $\mathbf{k}$ by

$$
\begin{aligned}
& \text { II }: T_{p} M \longrightarrow T_{p} \mathscr{H}, \\
& X \longrightarrow X+g(\ell, X) \mathbf{k} .
\end{aligned}
$$

The above mapping is well defined; that is, its image is in $T_{p} \mathscr{H}$. Indeed,

$$
\forall X \in T_{p} M, \quad g(\ell, \mathbf{I I}(X))=g(\ell, X) g(\ell, \mathbf{k})=0 .
$$

Observe that II leaves any vector in $T_{p} \mathscr{H}$ invariant and $\mathbf{I I}(\mathbf{k})=0$. Moreover, the definition of the projector II does not depend on the normalization of $\ell$ and $\mathbf{k}$ as long as they satisfy the relation (20). In other words, II is determined only by the foliation of the family $\left(\mathbf{S}_{t, u}\right)$ of $\mathscr{H}_{u}$ and not by any rescaling of $\ell$.

Consider a spacelike orthonormal frames field $E=$ $\left\{e_{1}, e_{2}\right\}$ on $\mathbf{S}_{t}$ of $\mathscr{H}$. Since $B(\ell, \ell)=0$, the expansion scalar field (also called null mean curvature) $\theta_{(\ell)}$ of $\mathscr{H}$ with respect to $\ell$ can be defined by

$$
\theta_{(\ell)}=B\left(e_{1}, e_{1}\right)+B\left(e_{2}, e_{2}\right)
$$

which is equivalent to trace $(B)$ and therefore it does not depend on the frame $E$. For a totally umbilical $\mathscr{H}$ this means that

$$
\theta_{(\ell)}=B\left(e_{1}, e_{1}\right)+B\left(e_{2}, e_{2}\right)=f\left\{h\left(e_{1}, e_{1}\right)+h\left(e_{2}, e_{2}\right)\right\}=2 f .
$$

Then, $\mathscr{H}$ is totally geodesic (also called minimal) if $\theta_{(\ell)}$ vanishes; that is,

$$
B\left(e_{1}, e_{1}\right)+B\left(e_{2}, e_{2}\right)=0
$$

2.2. Induced Extrinsic Structure Equations. Recall that Duggal and Bejancu [17, Chapter 4] used a screen distribution to obtain induced extrinsic objects of a lightlike hypersurface. Although we are not using any screen for $\mathscr{H}$, we do have a vector bundle TS of the 2-dimensional submanifolds of $M$. In order to use the extrinsic structure equations given in [17, Chapter 4], we replace the role of screen by the vector bundle $T S$ of $M$ which has the added advantage that it is obviously integrable. With this understanding, from (20) we have the following decomposition of $T M_{\mid \mathscr{H}}$ :

$$
T M_{\mid \mathscr{H}}=T \mathscr{H} \oplus_{\text {orth }} \operatorname{tr}(T \mathscr{H}),
$$

where $\operatorname{tr}(T \mathscr{H})=\{\mathbf{k}\}$ denotes a null transversal vector bundle of rank 1 over $\mathscr{H}$. Using the above decomposition and the second fundamental form $B$, we obtain the following extrinsic Gauss and Weingarten formulas [17, Chapter 4]:

$$
\begin{gathered}
\nabla_{X} Y=\mathscr{D}_{X} Y+B(X, Y) \mathbf{k}, \\
\nabla_{X} \mathbf{k}=-A_{\mathbf{k}} X+\tau(X) \mathbf{k}, \quad \forall X, Y \in \Gamma(T \mathscr{H}),
\end{gathered}
$$

where $A_{\mathbf{k}}$ is the shape operator on $T_{p} \mathscr{H}$ in $M, \tau$ is a 1 -form on $\mathscr{H}$, and $\mathscr{D}$ is the induced linear connection on a pair $(\mathscr{H},[\ell])$. By setting $Y=\ell$ in (28) the Weingarten map $\mathscr{W}_{\ell}$, defined by (6), will satisfy.

$$
\mathscr{D}_{X} \ell=\mathscr{W}_{\ell} X, \quad \forall X \in T \mathscr{H},
$$

with respect to the induced linear connection $\mathscr{D}$ on a pair $(\mathscr{H},[\ell])$. In general, $\mathscr{D}$ is not a Levi-Civita connection and it satisfies

$$
\begin{gathered}
\left(\mathscr{D}_{X} h\right)(Y, Z)=B(X, Y) \eta(Z)+B(X, Z) \eta(Y), \\
\forall X, Y, Z \in \Gamma\left(T \mathscr{H}_{\mid \mathcal{U}}\right)
\end{gathered}
$$

where $\eta(X)=g(X, \mathbf{k}) \quad \forall X \in \Gamma\left(T \mathscr{H}_{\mid \mathcal{U}}\right)$. Let $R$ and $\mathscr{R}$ denote the curvature tensors of the Levi-Civita connection $\nabla$ on $M$ and the induced linear connection $\mathscr{D}$ on $\mathscr{H}$, respectively. The Gauss-Codazzi equations are

$$
\begin{aligned}
& g(R(X, Y) Z, V)=h(\mathscr{R}(X, Y) Z, V), \quad \forall V \in T S . \\
& g(R(X, Y) Z, \ell)= h(\mathscr{R}(X, Y) Z, \ell) \\
&=\left(\nabla_{X} B\right)(Y, Z)-\left(\nabla_{Y} B\right)(X, Z) \\
&+B(Y, Z) \tau(X)-B(X, Z) \tau(Y) . \\
& g(R(X, Y) Z, \mathbf{k})=h(\mathscr{R}(X, Y) Z, \mathbf{k}) .
\end{aligned}
$$

The induced Ricci tensor of $(\mathscr{H}, h)$ is given by the following formula:

$$
\mathscr{R}(X, Y)=\operatorname{trace}\{Z \longrightarrow \mathscr{R}(X, Z) Y\}, \quad \forall X, Y \in \Gamma(T \mathscr{H}) .
$$

Since $\mathscr{D}$ on $\mathscr{H}$ is not a Levi-Civita connection, in general, Ricci tensor is not symmetric. Indeed, let $F=\left\{\ell, \mathbf{k}, e_{1}, e_{2}\right\}$ be a quasiorthonormal frame on $M$. Then, we obtain

$$
\begin{aligned}
\mathscr{R}(X, Y)= & h\left(\mathscr{R}\left(e_{1}, X\right) Y, e_{1}\right) \\
& +h\left(\mathscr{R}\left(e_{2}, X\right) Y, e_{2}\right)+h(\mathscr{R}(\ell, X) Y, \mathbf{k}) .
\end{aligned}
$$


Using Gauss-Codazzi equations and the first Bianchi identity we get

$$
\mathscr{R}(X, Y)-\mathscr{R}(Y, X)=2 d \tau(X, Y) .
$$

Also, the 1-form $\tau$ in (29) depends on the choice of the normal $\ell$. Therefore, we must require that $\mathscr{R}$ is symmetric (otherwise it has no geometric or physical meaning) and $\tau$ vanishes so that $A_{\mathbf{k}}$ is independent of the choice of the foliation $\left(\mathscr{H}_{u}\right)$. To fix this problem we assume the following known result.

Proposition 7 (see [17, page 99]). Let $(\mathscr{H}, h)$ be a null hypersurface of a semiRiemannian manifold $(M, g)$. If the induced Ricci tensor of $\mathscr{H}$ is symmetric then there exists a local null pair $\{\ell, \mathbf{k}: g(\ell, \mathbf{k})=-1\}$ such that the corresponding 1 form $\tau$ from (29) vanishes.

For a Ricci symmetric totally umbilical $\mathscr{H}(28)-(30)$ reduce to

$$
\begin{gathered}
\nabla_{X} Y=\mathscr{D}_{X} Y+f g(X, Y) \mathbf{k}, \\
\nabla_{X} \mathbf{k}=-A_{\mathbf{k}} X, \\
\left(\mathscr{D}_{X} h\right)(Y, Z)=f\{g(X, Y) \eta(Z)+g(X, Z) \eta(Z)\}, \\
\forall X, Y, Z \in \Gamma\left(T \mathscr{H}_{\mid \mathcal{U}}\right) .
\end{gathered}
$$

\section{Evolving Null Horizons}

Here we state the notion of an "Evolving Null Horizon," denoted by ENH, explain the implications of its conditions, construct some examples of ENHs, establish their relation with event and isolated horizons, and construct two physical models of an ENH of a black hole spacetime.

Definition 8. A null hypersurface $(\mathscr{H}, h)$ of a 4-dimensional spacetime $(M, g)$ is called an evolving null horizon $(\mathrm{ENH})$ if

(i) $\mathscr{H}$ is totally umbilical and may include a totally geodesic portion;

(ii) all equations of motion hold at $\mathscr{H}$ and stress energy tensor $T_{i j}$ is such that $-T_{j}^{i} \ell^{j}$ is future-causal for any future-directed null normal $\ell^{i}$.

For condition (i), it follows from Proposition 6 that $£_{\ell} h=$ $2 f h$ on $\mathscr{H}$; that is, $\ell$ is a conformal Killing vector $(\mathrm{CKV})$ of the metric $h$. If $\mathscr{H}$ includes a totally geodesic portion, then on its portion $f$ vanishes and $\ell$ reduces to a Killing vector. This does not necessarily imply that $\ell$ is a CKV (or Killing vector) of the full metric $g$. The shear tensor $\sigma$ is given by

$$
\sigma=\mathfrak{E}_{\ell} h-\frac{1}{2} \theta_{(\ell)} h=\left(2 f-\frac{1}{2} \theta_{(\ell)}\right) h .
$$

The energy condition of (ii) requires that $R_{i j} \ell^{i} \ell^{j}$ is nonnegative for any $\ell$; which implies (see Hawking and Ellis $[19$, page 95]) that $\theta_{(\ell)}$ monotonically decreases in time along $\ell$, that is, $M$ obeys the null convergence condition. Condition (i) also implies that we have two classes of ENHs, namely, generic ENH ( $f$ does not vanish on $\mathscr{H}$ ) and nongeneric ENH for which $f$ may vanish on a possible totally geodesic portion of $\mathscr{H}$. The metric on a generic ENH will be time-dependent and $\theta_{(\ell)}$ will not vanish on $\mathscr{H}$. In the case of a non-generic ENH, $\theta_{(\ell)}$ may eventually vanish on its totally geodesic portion for which its transformed metric will be time-independent. We give examples of both classes.

3.1. Mathematical Model. Let $(\mathscr{H}, h)$ be a member of the family $\left(\mathscr{H}_{u}\right)$ of null hypersurfaces of a spacetime $(M, g)$ whose metric $g$ is given by (12). With respect to each $\mathbf{S}_{t}$, the shift vector $U$ can be expressed as

$$
U=\alpha \mathbf{s}-V, \quad \alpha=\mathbf{s} \cdot U, \quad V \in T_{p}\left(\mathbf{S}_{t}\right) .
$$

Using (13), (17), and (38) we obtain

$$
\ell=\mathbf{t}+V+(\lambda-\alpha) \mathbf{s} .
$$

To relate, above decomposition of $\ell$ with the conformal function $f$ of the totally umbilical condition (8), we choose $\lambda-\alpha=f$ on $\mathscr{H}$, which means that a portion of $\mathscr{H}$ may be totally geodesic if and only if $\lambda=\alpha$ on that portion. Thus,

$$
\ell \stackrel{\mathscr{H}}{=} \mathbf{t}+V+f \mathbf{s}
$$

Consider a coordinate system $\left(x^{A}\right)=\left(t, x^{2}, x^{3}\right)$ on $(\mathscr{H}, h)$ defined by $\left\{x^{1}=\right.$ constant $\}$. Then, the degenerate metric $h$ is

$$
h=h_{A B} d x^{A} d x^{B}=h_{t t} d t^{2}+2 h_{t k} d t d x^{k}+h_{k m} d x^{k} d x^{m},
$$

where $2 \leq k, m \leq 3$, and

$$
h_{t t}=V^{k} V_{k}+f^{2}, \quad h_{t k}=U_{k}=\alpha \mathbf{s}_{k}-V_{k}=-V_{k} .
$$

Observe that one can also take $x^{2}$ or $x^{3}$ constant. Assume that $(\mathscr{H}, h)$ is proper totally umbilical in $(M, g)$ and $M$ obeys the null convergence condition, with respect to each futuredirected null normal of this family $\left(\mathscr{H}_{u}\right)$. Then, a member ( $\mathscr{H}, h, x^{1}=$ constant) of this family is a model of a generic $\mathrm{ENH}$ whose degenerate metric is given by (41).

Nongeneric ENH. Consider a null hypersurface $\mathscr{H}=\Sigma \cup \Delta$ such that $\Sigma$ and $\Delta$ are null proper totally umbilical and totally geodesic portions of $\mathscr{H}$ and $M$ obeys the null convergence condition. Thus, as per Definition $8, \mathscr{H}$ is an ENH which includes a totally geodesic portion $(\Delta, q)$, where $q$ denotes its metric. Since $\theta_{(\ell)}$ monotonically decreases in time along $\ell$ and for this case $f$ can vanish on $\Delta$, a state may reach at a time when $\theta_{(\ell)}$ vanishes on the portion $\Delta$ of $\mathscr{H}$ and by putting $f=0$ in the metric (41) we recover the metric $q$ of $\Delta$ given by

$$
q=q_{A B} d x^{A} d x^{B}=q_{k m}\left(d x^{k}-V^{k} d t\right)\left(d x^{m}-V^{m} d t\right),
$$

where $\mathfrak{E}_{\ell} q=0$ and

$$
\begin{aligned}
& \alpha \stackrel{(\mathscr{H}, q)}{=} \lambda \text { is constant on }(\mathscr{H}, q) \text {, } \\
& \ell^{(\mathscr{H}, q)} \stackrel{\mathbf{t}}{=}+V \text {. }
\end{aligned}
$$


For this case the transformed coordinate system $\left(x^{A}\right)$ is stationary with respect to the hypersurface $(\mathscr{H}, q)$ as its metric (43) is time-independent. In other words, the location of $\mathbf{S}_{t}$ is fixed as $t$ varies. Moreover,

$$
\begin{aligned}
\left(\left(x^{A}\right) \text { stationary w.r.t. }(\mathscr{H}, q)\right) & \Longleftrightarrow \frac{\partial u}{\partial t}=0 \\
& \Longleftrightarrow \mathbf{t} \in T(\mathscr{H}) .
\end{aligned}
$$

At this state of transition, Raychaudhuri equation (2) implies that shear also vanishes. Consequently, the two conditions of the definition of a totally geodesic evolving null horizon $(\mathscr{H}, q)$ reduce to conditions (2) and (3) of a nonexpanding horizon $(\mathrm{NEH})$. In case $(\mathscr{H}, q)$ is a null horizon of some black hole, then the vector $V \in T_{p}\left(\mathbf{S}_{t}\right)$ is called the surface velocity of the black hole (see Damour [20]). We present the following mathematical model.

Monge Null Hypersurfaces. Consider a smooth function $F$ : $\Omega \rightarrow \mathbf{R}$, where $\Omega$ is an open set of $\mathbf{R}^{3}$. Then a hypersurface $\mathscr{H}$ of $\mathbf{R}_{1}^{4}$ is called a Monge hypersurface [17, page 129] given by the equation

$$
t=F(x, y, z)
$$

where $(t, x, y, z)$ are the standard Minkowskian coordinates with origin $\mathbf{0}$. The scalar $u$ generates a family of Monge hypersurfaces $\left(\mathscr{H}_{u}\right)$ as the level sets of $u$ and is given by

$$
u(t, x, y, z)=F-t+b,
$$

where $b$ is a constant and we take $\mathscr{H}_{b}=\mathscr{H}$ a member of the family $\left(\mathscr{H}_{u}\right) . \nabla_{i} u=\left(-1, F_{x}^{\prime}, F_{y}^{\prime}, F_{z}^{\prime}\right) . \mathscr{H}$ is null if and only if $F$ is a solution of the following partial differential equation:

$$
\left(F_{x}^{\prime}\right)^{2}+\left(F_{y}^{\prime}\right)^{2}+\left(F_{z}^{\prime}\right)^{2}=1 \text {. }
$$

From (19) the null normal $\ell$ of $\mathscr{H}$ is

$$
\ell^{i}=e^{\rho}\left(1, F_{x}^{\prime}, F_{y}^{\prime}, F_{z}^{\prime}\right), \quad \ell_{i}=e^{-\rho}\left(-1, F_{x}^{\prime}, F_{y}^{\prime}, F_{z}^{\prime}\right) .
$$

The components of the transversal vector field $\mathbf{k}$ can be taken as

$$
\begin{aligned}
& \mathbf{k}^{i}=\frac{1}{2} e^{\rho}\left(-1,-F_{x}^{\prime},-F_{y}^{\prime},-F_{z}^{\prime}\right), \\
& \mathbf{k}_{i}=\frac{1}{2} e^{-\rho}\left(1,-F_{x}^{\prime},-F_{y}^{\prime},-F_{z}^{\prime}\right),
\end{aligned}
$$

so that $h(\ell, \mathbf{k})=-1$. The corresponding base vectors, say $\left\{W_{1}, W_{2}\right\}$, of $\mathbf{S}_{\mathbf{t}}$ are

$$
\left\{W_{1}=F_{z}^{\prime} \frac{\partial}{\partial x}-F_{x}^{\prime} \frac{\partial}{\partial z} ; W_{2}=F_{z}^{\prime} \frac{\partial}{\partial y}-F_{y}^{\prime} \frac{\partial}{\partial z}\right\},
$$

where we take $F_{z}^{\prime} \neq 0$ on $\mathscr{H}$. Then, the 1 -form $\tau$ in (29) vanishes. Indeed, $\tau(X)=g\left(\nabla_{X} \mathbf{k}, \ell\right)=-(1 / 2) g\left(\nabla_{X} \ell, \ell\right)=0$. Therefore, it is quite straightforward (see [17, page 121]) that the Ricci tensor of the linear connection $\mathscr{D}$ on this Monge hypersurface $\mathscr{H}$ is symmetric. Consequently, our extrinsic objects on null Monge hypersurfaces are independent of the choice of a family $\left(\mathscr{H}_{u}\right)$. To find the expansion $\theta_{(\ell)}$ of $\mathscr{H}$ we use the base vectors (51) to construct the following orthonormal basis $\left\{e_{1}, e_{2}\right\}$ of $\mathbf{S}_{\mathbf{t}}$ :

$$
\begin{gathered}
e_{1}=\frac{1}{\left\|W_{1}\right\|} W_{1} ; \\
e_{2}=\frac{1}{\left|F_{z}^{\prime}\right|\left\|W_{1}\right\|}\left\{\left\|W_{1}\right\|^{2} W_{2}-g\left(W_{1}, W_{2}\right) W_{1}\right\} .
\end{gathered}
$$

Then by direct calculations we obtain

$$
\begin{aligned}
B\left(e_{1}, e_{1}\right)+B\left(e_{2}, e_{2}\right) \\
=\frac{1}{\left(F_{z}^{\prime}\right)^{2} h_{11}} \\
\quad \times\left\{\left(\left(F_{z}^{\prime}\right)^{2}+\left(h_{12}\right)^{2}\right) B_{11}-2 h_{11} h_{12} B_{12}+\left(h_{11}\right)^{2} B_{22}\right\},
\end{aligned}
$$

where we put $h_{\alpha \beta}=h\left(W_{\alpha}, W_{\beta}\right)$ and $B_{\alpha \beta}=B\left(W_{\alpha}, W_{\beta}\right), \alpha, \beta \epsilon$ $\{1,2\}$. Taking into account that

$$
h_{11}=1-\left(F_{y}^{\prime}\right)^{2}, \quad h_{12}=F_{x}^{\prime} F_{y}^{\prime}, \quad h_{22}=1-\left(F_{x}^{\prime}\right)^{2},
$$

we finally (see pages 118-132 in [17] for some missing details) obtain

$$
\theta_{(\ell)}=B\left(e_{1}, e_{1}\right)+B\left(e_{2}, e_{2}\right)=-\frac{1}{\left(F_{z}^{\prime}\right)^{2} h_{11}}\left(F_{x x}^{\prime \prime}+F_{y y}^{\prime \prime}+F_{z z}^{\prime \prime}\right) .
$$

Consider a family $\left(\mathscr{H}_{u}\right)$ of null Monge hypersurfaces of $\mathbf{R}_{1}^{4}$ whose each member $(\mathscr{H}, h)$ obeys the null convergence condition; that is, $\theta_{(\ell)}$ monotonically decreases in time along $\ell$. Thus, it follows from $(26)$ and $(55)$ that $(\mathscr{H}, h)$ may have a totally geodesic portion, say $\Delta$, if and only if a state reaches when $\theta_{(\ell)}$ vanishes and at that state $F$ satisfies the Laplace equation:

$$
F_{x x}^{\prime \prime}+F_{y y}^{\prime \prime}+F_{z z}^{\prime \prime}=0
$$

which means that $F$ is harmonic on $\Delta$. As explained in Section 2, take a family $\left(\mathscr{H}_{u}\right)$ of null Monge hypersurfaces of $\mathbf{R}_{1}^{4}$ whose each member $(\mathscr{H}, h)$ is totally umbilical $(B=2 f h)$. Then, as per (24) and (55), we have

$$
\theta_{(\ell)}=-\frac{1}{\left(F_{z}^{\prime}\right)^{2} g_{11}}\left(F_{x x}^{\prime \prime}+F_{y y}^{\prime \prime}+F_{z z}^{\prime \prime}\right)=2 f,
$$

which implies that $F$ is harmonic on $\Delta$ if and only if $f$ vanishes on $\Delta$. Thus, there exists a model of a class of Monge null hypersurfaces which is union of a family of generic ENHs and its totally geodesic null portions.

Generic ENHs. Now we show by the following example that there are some Monge ENHs which are generic; that is, they do not evolve into a totally geodesic ENH. 
Example 9 (see [16]). Let $\left(\Lambda_{0}^{3}\right)_{u}$ be a family of Monge null cones in $\mathbf{R}_{1}^{4}$ given by

$$
t=F(x, y, z)=r=\sqrt{x^{2}+y^{2}+z^{2}},
$$

where $(t, x, y, z)$ are the Minkowskian coordinates with origin $\mathbf{0}$. Exclude $\mathbf{0}$ to keep each null cone smooth. Let $\Lambda_{0}^{3}$ be a member at the level $u=b \neq 0$. Then, the scalar $u$ generates a family of Monge null cones $\left(\left(\Lambda_{0}^{3}\right)_{u}\right)$ given by

$$
u(t, x, y, z)=r-t+b, \quad \text { with } t=\sqrt{x^{2}+y^{2}+z^{2}}
$$

$\nabla_{i} u=(-1, x / t, y / t, z / t)$. Thus, the components of the null normal to $\Lambda_{0}^{3}$ are

$$
\ell^{i}=e^{\rho}\left(1, \frac{x}{t}, \frac{y}{t}, \frac{z}{t}\right), \quad \ell_{i}=e^{-\rho}\left(-1, \frac{x}{t}, \frac{y}{t}, \frac{z}{t}\right) .
$$

Since $\ell$ is the position vector field for the above construction of the set of null cones, it follows from the Gauss equation (28) that

$$
\nabla_{X} \ell=\mathscr{D}_{X} \ell=u X, \quad \forall X \in\left(\Lambda_{0}^{3}\right)_{u},
$$

where $u \neq 0$ as $\mathbf{0}$ is excluded. Then, using the Weingarten map equation (6), we obtain

$$
\mathscr{W}_{\ell} X=u X=f X, \quad \forall \text { non-null } X \in\left(\Lambda_{0}^{3}\right)_{u} .
$$

Hence, by (9) we conclude that any member of the set of null cones $\left(\left(\Lambda_{0}^{3}\right)_{u \neq 0}\right)$ is proper totally umbilical with the conformal function $f=u \neq 0$. The unit timelike and spacelike normals $\mathbf{n}$ and $\mathbf{s}$ in the normalized expression of $\ell$ are

$$
\mathbf{n}^{i}=(1,0,0,0), \quad \mathbf{s}^{i}=\left(0, \frac{x}{t}, \frac{y}{t}, \frac{z}{t}\right) .
$$

Therefore, it follows from (19) that $\lambda=f+\alpha=e^{\rho}$. To calculate the expansion $\theta_{(\ell)}=2 f$, we use (57) for $F=r$ and obtain

$$
\begin{aligned}
\theta_{(\ell)} & =-\frac{1}{\left(r_{z}^{\prime}\right)^{2} h_{11}}\left(r_{x x}^{\prime \prime}+r_{y y}^{\prime \prime}+r_{z z}^{\prime \prime}\right) \\
& =-\frac{2 t^{3}}{z^{2}\left(t^{2}-y^{2}\right)}=-\frac{2 t^{3}}{z^{2}\left(x^{2}+z^{2}\right)}=2 f \neq 0 .
\end{aligned}
$$

Since $f$ does not vanish on $\mathscr{H}$, subject to the null convergence condition, the above is an example of an ENH which does not evolve into an event or isolated horizon.

\section{Two Physical Models Having an ENH of a Black Hole Spacetime}

Nongeneric Model. Let $(M, g)$ be a spacetime which admits a totally geodesic null hypersurface $(\mathscr{H}, q, \ell)$, where its degenerate metric $q$ is the pull back of $g$ and $\ell$ is its future-directed null normal defined in some open subset of $M$ around $\mathscr{H}$ (see details in Section 1). Then, we know that $\mathfrak{E}_{\ell} q=0$; that is, $\ell$ is Killing with respect to the metric $q$, which is not necessarily Killing with respect to full metric $g$. Consider a metric conformal transformation defined by

$$
G=\Omega^{2} g,
$$

where $\Omega$ is a scalar function on $M$ and $(M, G)$ is its conformal spacetime manifold. Since the causal structure and null geodesics are invariant under a conformal transformation, as discussed in Section 2, we have a family of null hypersurfaces $\left(\mathscr{H}_{u}\right)$ in $(M, G)$ with its corresponding family of induced metrics $\left(h_{u}\right)$ such that each one is conformally related to $q$ by the following transformation:

$$
h_{u}=\Omega_{u}^{2} q,
$$

for some value of the parameter $u$ of scalar functions $\left(\Omega_{u}\right)$ on $\mathscr{H}$ and $\ell$ is in the part of $(M, G)$ foliated by this family such that at each point in this region, $\ell$ is a future null normal to $\mathscr{H}_{u}$ for some value of $u$. Set $(\mathscr{H}, h, \Omega)$ as a member of $\left(\mathscr{H}_{u}\right)$ for some $u$. It is easy to show that $\mathfrak{E}_{\ell} h=2 \ell(\ln (\Omega)) h$; that is, $\ell$ is conformal Killing with respect to the metric $h$, which is the image of the corresponding Killing null vector with respect to the metric $q$. Thus, as per Proposition $6,\left(\left(\mathscr{H}_{u}\right),\left(h_{u}\right)\right)$ is a family of totally umbilical null hypersurfaces of $(M, G)$ such that the second fundamental form $B$ of its each member $(\mathscr{H}, h, \Omega)$ satisfies

$$
B(X, Y)=\ell(\ln (\Omega)) h(X, Y), \quad \forall X, Y \in T(\mathscr{H}),
$$

where the conformal function $f=\ell(\ln (\Omega))$. Thus, subject to condition (ii) of Definition 8 each member of $\left(\mathscr{H}_{u}\right)$ is an ENH of $(M, G)$ for some value of $u$. Suppose the null hypersurface $(\mathscr{H}, q)$ of $(M, g)$ is an event or an isolated horizon such that (65) and (66) hold. Then, the family $\left(\left(\mathscr{H}_{u}\right),\left(h_{u}\right)\right)$ of ENHs evolves into the corresponding event or isolated horizon $(\mathscr{H}, q)$ when $G \rightarrow g$; that is, $\Omega \rightarrow 1$. The following is an example of such an ENH of a BH spacetime which evolves into a weakly isolated horizon.

Example 10. Let $(M, g)$ be Einstein static universe with metric $g$ given by

$$
g=-d t^{2}+d r^{2}+r^{2}\left(d \theta^{2}+\sin ^{2} \theta d \phi^{2}\right),
$$

where $(t, r, \theta, \phi)$ is a spherical coordinate system. This metric is singular at $r=0$ and $\sin \theta=0$. We, therefore, choose the ranges $0<r<\infty, 0<\theta<\pi$, and $0<\phi<2 \pi$ for which it is a regular metric. Take two null coordinates $v$ and $w$, with respect to a pseudo-orthonormal basis, such that $v=t+r$ and $w=t-r(v \geq w)$. We get the following transformed metric:

$$
g=-d v d w+\left(\frac{v-w}{2}\right)^{2}\left(d \theta^{2}+\sin ^{2} \theta d \phi^{2}\right),
$$

where $-\infty<v, w<\infty$. The absence of the terms $d v^{2}$ and $d w^{2}$ in (69) implies that the hypersurfaces $\{w=$ constant $\}$ and $\{v=$ constant $\}$ are null future and past-directed hypersurfaces, respectively, since $w_{; a} w_{; b} \eta^{a b}=0=v_{; a} v_{; b} \eta^{a b}$. Consider a future-directed null hypersurface $(\mathscr{H}, q, w=$ constant $)$ of 
$(M, g)$. It is easy to see that the degenerate metric $q$ of this $\mathscr{H}$ is given by

$$
q=\left(\frac{v-w}{2}\right)^{2}\left(d \theta^{2}+\sin ^{2} \theta d \phi^{2}\right), \quad w=\text { constant }
$$

which is time-independent and has topology $R \times S^{2}$. Therefore, $\mathfrak{f}_{\ell} q=0$; that is, $\mathscr{H}, q, w=$ constant is totally geodesic in $(M, g)$ which further implies that its future-directed null normal $\ell$ has vanishing expansion, $\theta_{(\ell)}=0$. Thus, subject to the energy condition (3) of Definition 1, this $\mathscr{H}$ is an $\mathrm{NEH}$ which (see Ashtekar-Fairhust-Krishnan [9]) can be a weakly isolated horizon.

Consider $(M, G)$ the de-Sitter spacetime [19] which is of constant positive curvature and is topologically $R^{1} \times S^{3}$ with its metric $G$ given by

$$
G=-d t^{2}+a^{2} \cosh ^{2}\left(\frac{t}{a}\right)\left\{d r^{2}+r^{2}\left(d \theta^{2}+\sin ^{2} \theta d \phi^{2}\right)\right\},
$$

where $a$ is nonzero constant. Its spacial slices ( $t=$ constant) are 3 -spheres. Introducing a new timelike coordinate $\tau=$ $2 \arctan (\exp (t / 2))-(\pi / 2)$, we get

$$
G=a^{2} \cosh ^{2}\left(\frac{\tau}{a}\right) g, \quad\left(-\frac{\pi}{2}<\tau<\frac{\pi}{2}\right),
$$

where the metric $g$ is given by (69). Thus, the de-Sitter spacetime $(M, G)$ is locally conformal to the Einstein static universe $(M, g)$ with the conformal function $\Omega=a \cosh (\tau / a)$. As discussed above, we have a family of null hypersurfaces $\left(\left(\mathscr{H}_{u}\right),\left(h_{u}\right)\right)$ in $(M, G)$ whose metrics $\left(h_{u}\right)$ are conformally related to the metric $q$ by the transformation:

$$
\begin{aligned}
h_{u}= & a^{2} \cosh ^{2}\left(\frac{\tau}{a}\right) q=a^{2} \cosh ^{2}\left(\frac{\tau}{a}\right) \\
& \times\left\{\left(\frac{v-w}{2}\right)^{2}\left(d \theta^{2}+\sin ^{2} \theta d \phi^{2}\right)\right\},
\end{aligned}
$$

for some value of the parameter $u=\tau$ of scalar functions $\left(\Omega_{\tau}\right)$ on $\mathscr{H}$, where $w=$ constant and $q$ is the metric of the null hypersurface $(\mathscr{H}, q)$ of $(M, g)$. Set $(\mathscr{H}, h, \ell)$ as a member of this family $\left(\left(\mathscr{H}_{u}\right),\left(h_{u}\right)\right)$. Then, the following holds:

$$
\begin{gathered}
\mathfrak{E}_{\ell} h=2 a \ell\left(\ln \left(\cosh \left(\frac{\tau}{a}\right)\right) h\right), \\
B(X, Y)=f h(X, Y)=a \ell\left(\ln \left(\cosh \left(\frac{\tau}{a}\right)\right) h(X, Y)\right), \\
\forall X, Y \in T(\mathscr{H}) .
\end{gathered}
$$

Therefore, we have a family of totally umbilical timedependent null hypersurfaces $\left(\left(\mathscr{H}_{u}\right),\left(h_{u}\right)\right)$ of $(M, G)$. Subject to the condition (ii) of Definition 8 this is a family of ENHs of the de-Sitter spacetime $(M, G)$ which can evolve into a WIH when $G \rightarrow g$ at the equilibrium state of $M$.

Generic Model. Consider a spacetime $(M, g)$ with Schwarzschild metric:

$$
g=-A(r)^{2} d r^{2}+A(r)^{-2} d r^{2}+r^{2}\left(\sin ^{2} \theta d \phi^{2}+d \theta^{2}\right),
$$

where $A(r)^{2}=1+2 m / r$ and $m$ and $r$ are the mass and the radius of a spherical body. Construct a 2-dimensional submanifold $\mathbf{S}$ of $M$ as an intersection of a timelike hypersurface $r=$ constant with a spacelike hypersurface $t=$ constant. Choose at each point of $\mathbf{S}$ a null direction perpendicular in S, smoothly depending on the foot-point. There are two such possibilities: the ingoing and the outgoing radical directions. Let $\mathscr{H}$ be the union of all geodesics with the chosen (say outgoing) initial direction. This is a submanifold near $\mathbf{S}$. Moreover, the symmetry of the situation guarantees that $\mathscr{H}$ is a submanifold everywhere, except at points where it meets the centre of symmetry. It is very easy to verify that $\mathscr{H}$ is a totally umbilical null hypersurface of $M$. Since $\mathscr{H}$ does not include the points where it meets the centre of symmetry, it is proper totally umbilical in $M$; that is, $\mathscr{H}$ does not evolve into a totally geodesic portion. Therefore, subject to condition (ii) of Definition 8 and considering $\mathscr{H}$ a member of the family $\left(\mathscr{H}_{u}\right)$ we have a physical model of generic ENHs of the black hole Schwarzschild spacetime $(M, g)$.

\section{Discussion}

In this paper, we introduced a quasilocal definition of a family of null hypersurfaces called "evolving null horizons (ENH)" of a spacetime manifold. This definition uses some basic results taken from the differential geometry of totally umbilical hypersurfaces as opposed to present day use of totally geodesic geometry for event and isolated horizons. The first part of this paper includes enough background information on the event, three types of isolated and dynamical horizons, with a brief on their respective use in the study of black hole spacetimes, and need for introducing evolving null horizons.

The rest of this paper is focused on a variety of examples to justify the existence of ENHs and in some cases relate them with event and isolated horizons of a black hole spacetime. Thus, we have shown that an ENH describes the geometry of the null surface of a dynamical spacetime, in particular, a $\mathrm{BH}$ spacetime that also can be evolved into an event or isolated horizon at the equilibrium state of a landing spacetime. There is ample scope of further study on geometric/physical properties of ENHs. An input of the interested readers on what is presented so far is desired before one starts working on properties of ENHs, in particular reference to the null version of some known results on dynamical horizons. As is the case of introducing any new concept, there may be several questions on what we have presented in this paper. At this point in time, the following two fundamental issues need to be addressed.

We know that an event horizon always exists in black hole asymptotically flat spacetimes under a weak cosmic censorship condition and isolated horizons are precisely meant to model specifically quasilocal equilibrium regimes by offering a precise geometric approximation. Since so far we only have two specific models of an ENH in a black hole spacetime, one may ask. Does there always exist an ENH in a black hole spacetime?

Secondly, since an ENH $\mathscr{H}$ comes with an assigned foliation of null hypersurfaces its unique existence is questionable. Therefore, one may ask. Is there a canonical or unique choice of an evolving null horizon? 
Interested readers are invited for an input on these two and any other issues.

Finally, it is well-known that the rich geometry of totally umbilical submanifolds has a variety of uses in the world of mathematics and physics. Besides the needed study on the properties of evolving null horizons in black hole theory, further study on the mathematical theory of this paper alone has ample scope to a new way of research on the geometry of totally umbilical null hypersurfaces in a general semiRiemannian and for physical applications a spacetime manifold.

\section{Conflict of Interests}

The author declares that there is no conflict of interests regarding the publication of this paper.

\section{References}

[1] S. W. Hawking, "The event horizons," in Black Holes, C. DeWitt and B. DeWitt, Eds., Norht Holland, Amsterdam, The Netherlands, 1972.

[2] P. Hájíček, "Exact models of charged black holes. I. Geometry of totally geodesic null hypersurface," Communications in Mathematical Physics, vol. 34, pp. 37-52, 1973.

[3] P. Hájíček, "Can outside fields destroy black holes?” Journal of Mathematical Physics, vol. 15, pp. 1554-1558, 1974.

[4] P. Hájíček, "Stationary electrovacuum spacetimes with bifurcate horizons," Journal of Mathematical Physics, vol. 16, pp. 518-522, 1975.

[5] A. Ashtekar, C. Beetle, and S. Fairhurst, "Isolated horizons: a generalization of black hole mechanics," Classical and Quantum Gravity, vol. 16, no. 2, pp. L1-L7, 1999.

[6] G. J. Galloway, "Maximum principles for null hypersurfaces and null splitting theorems," Annales Henri Poincaré, vol. 1, no. 3, pp. 543-567, 2000.

[7] P. T. Chruściel, "Recent results in mathematical relativity," Classical Quantum Gravity, pp. 1-17, 2005.

[8] A. Ashtekar and B. Krishnan, "Isolated and dynamical horizons and their applications," Living Reviews in Relativity, vol. 7, pp. 1-91, 2004.

[9] A. Ashtekar, S. Fairhurst, and B. Krishnan, "Isolated horizons: hamiltonian evolution and the first law," Physical Review D, vol. 62, no. 10, Article ID 104025, 29 pages, 2000.

[10] A. Ashtekar, C. Beetle, and J. Lewandowski, "Geometry of generic isolated horizons," Classical and Quantum Gravity, vol. 19, no. 6, pp. 1195-1225, 2002.

[11] J. Lewandowski, "Spacetimes admitting isolated horizons," Classical and Quantum Gravity, vol. 17, no. 4, pp. L53-L59, 2000.

[12] E. Gourgoulhon and J. L. Jaramillo, "A $3+1$ perspective on null hypersurfaces and isolated horizons," Physics Reports, vol. 423, no. 4-5, pp. 159-294, 2006.

[13] S. A. Hayward, "General laws of black-hole dynamics," Physical Review D, vol. 49, no. 12, pp. 6467-6474, 1994.

[14] A. Ashtekar and B. Krishnan, "Dynamical horizons: energy, angular momentum, fluxes, and balance laws," Physical Review Letters, vol. 89, no. 26, Article ID 261101, 4 pages, 2002.

[15] A. Ashtekar and B. Krishnan, "Dynamical horizons and their properties," Physical Review D, vol. 68, no. 10, Article ID 104030, 25 pages, 2003.
[16] K. L. Duggal, "Foliations of lightlike hypersurfaces and their physical interpretation," Central European Journal of Mathematics, vol. 10, no. 5, pp. 1789-1800, 2012.

[17] K. L. Duggal and A. Bejancu, Lightlike Submanifolds of SemiRiemannian Manifolds and Applications, vol. 364 of Mathematics and Its Applications, Kluwer Academic Publishers, Dordrecht, The Netherlands, 1996.

[18] K. L. Duggal and D. H. Jin, Null Curves and Hypersurfaces of Semi-Riemannian Manifolds, World Scientific, Hackensack, NJ, USA, 2007.

[19] S. W. Hawking and G. F. R. Ellis, The Large Scale Structure of Space-Time, Cambridge Monographs on Mathematical Physics, no. 1, Cambridge University Press, London, UK, 1973.

[20] T. Damour, "Black-hole eddy currents," Physical Review D, vol. 18 , no. 10 , pp. $3598-3604,1978$. 


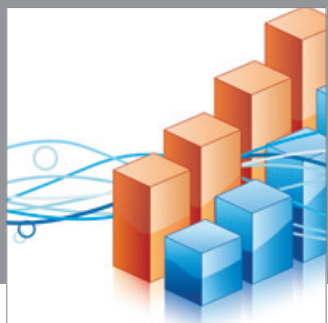

Advances in

Operations Research

mansans

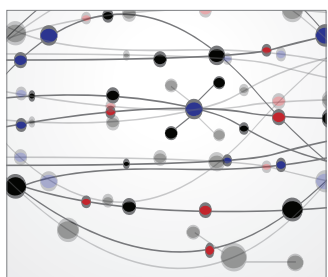

The Scientific World Journal
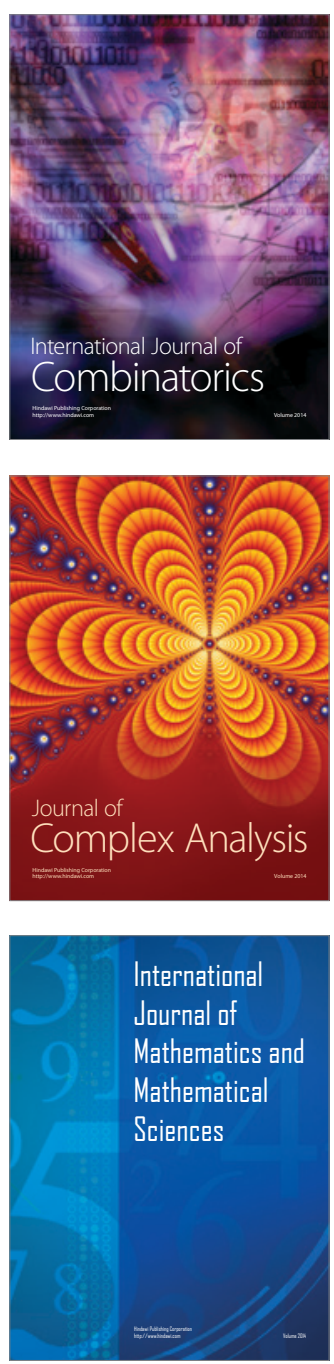
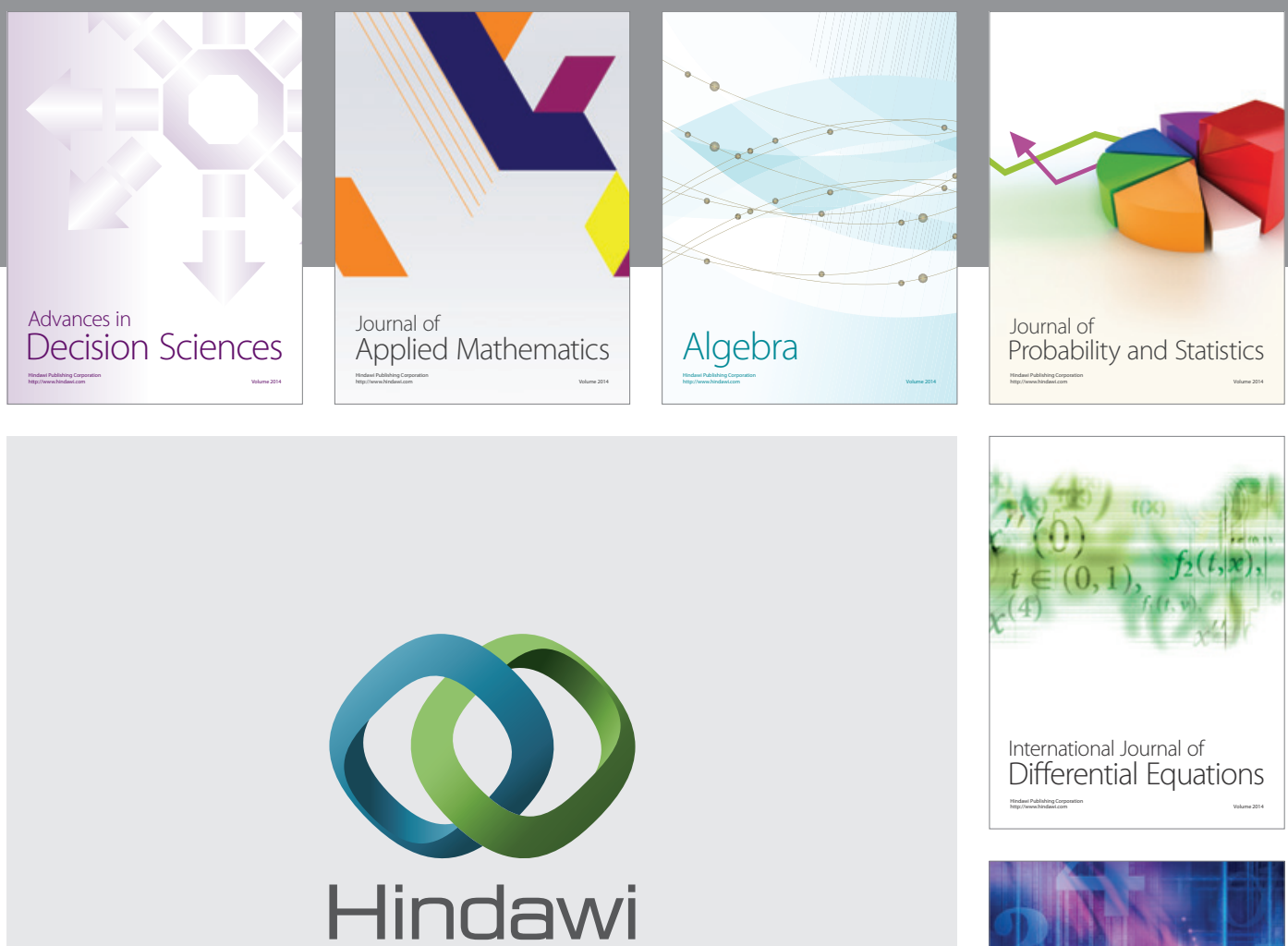

Submit your manuscripts at http://www.hindawi.com
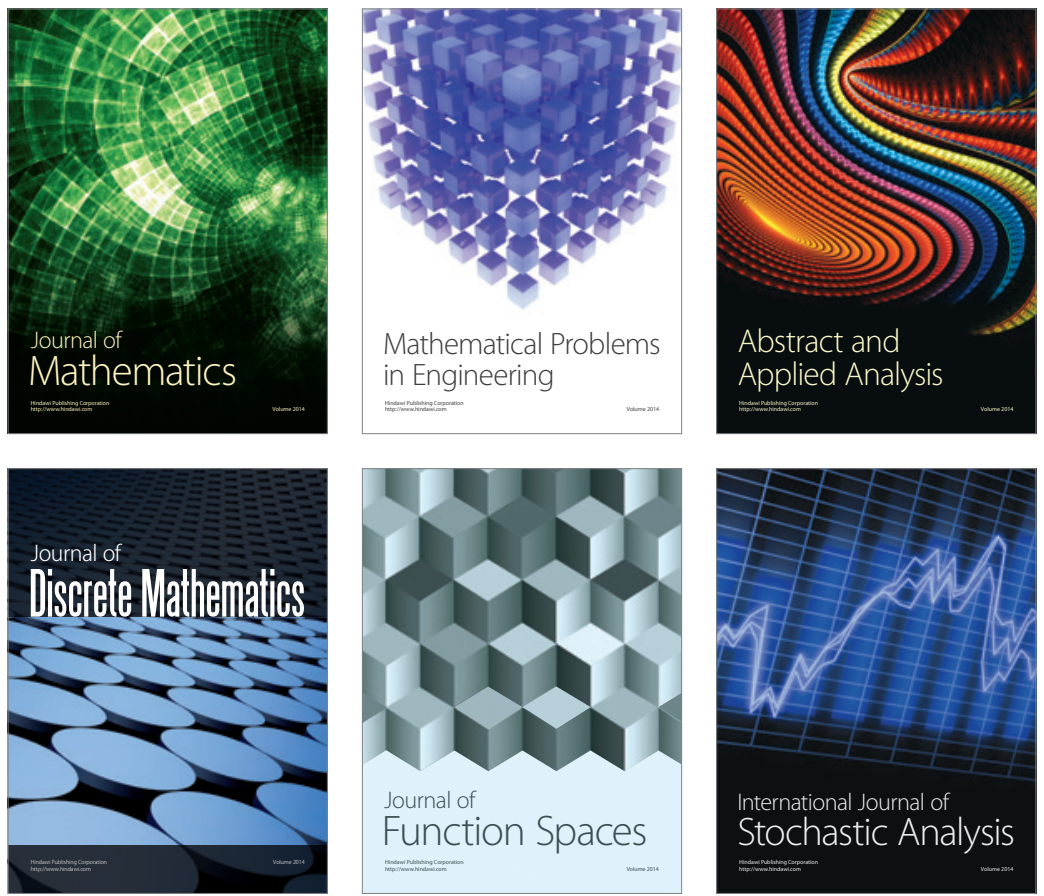

Journal of

Function Spaces

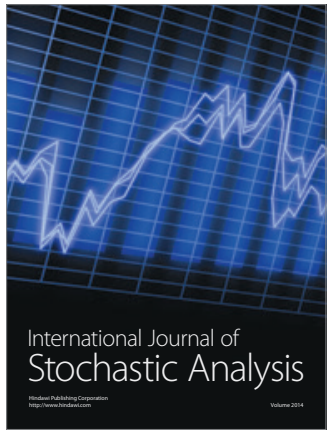

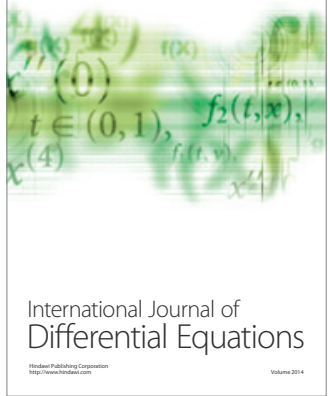
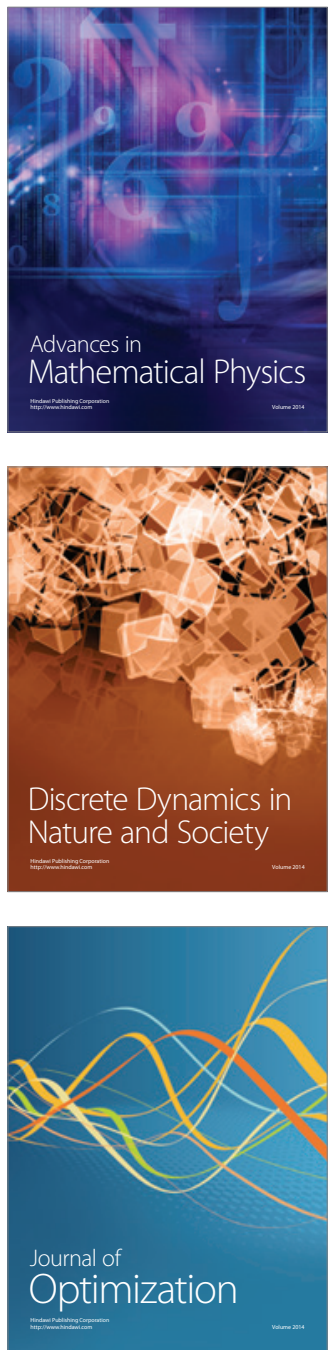